\title{
Speckle in photoacoustic tomography
}

\section{Li Li, Lihong V. Wang}

Li Li, Lihong V. Wang, "Speckle in photoacoustic tomography," Proc. SPIE 6086, Photons Plus Ultrasound: Imaging and Sensing 2006: The Seventh Conference on Biomedical Thermoacoustics, Optoacoustics, and Acoustooptics, 60860Y (6 March 2006); doi: 10.1117/12.646357

SPIE. Event: SPIE BiOS, 2006, San Jose, California, United States 


\title{
Speckle in Photoacoustic Tomography
}

\author{
Li Li and Lihong V. Wang* \\ Optical Imaging Laboratory, Department of Biomedical Engineering, \\ Texas A\&M University, College Station, Texas 77843-3120
}

\begin{abstract}
Photoacoustic tomography is emerging as a promising imaging modality for various biomedical applications. Unlike traditional ultrasound imaging that is plagued by strong speckle artifacts, no obvious speckle has so far been observed in photoacoustic images. We systematically studied the reason for this lack of speckle in photoacoustic tomography based on speckle contrast. Theoretical explanations were validated by simulation. The results here can serve as a basis for developing specific applications, such as tissue characterization, using photoacoustic methods.
\end{abstract}

Keywords: Speckle, Photoacoustic Tomography, Thermoacoustic Tomography, Linear System, Tissue Characterization

\section{INTRODUCTION}

First discovered by Bell in 1880, photoacoustic effect names a phenomenon where acoustic waves are produced by absorbing objects that are undergoing rapid electromagnetic illumination. Researchers have exploited its application with photoacoustic spectroscopy ${ }^{1}$. Recently, a technique called photoacoustic tomography (PAT) has emerged as a promising non-invasive imaging tool for various biomedical investigations. ${ }^{2,3,4}$ To be more specific, people tend to label the method thermoacoustic tomography when the excitation source is in the radiofrequency band and to call it photoacoustic tomography when the heating source is a pulsed laser. Several mechanisms responsible for the generation of photoacoustic waves were studied by Gusev ${ }^{5}$. Among them, thermo-elastic expansion is believed to be the most important in PAT. Ultrasound detectors are placed, or scanned, around an object to collect outgoing acoustic signals, which are later used to reconstruct the distribution of the initial acoustic pressure, or absorption coefficients under assumptions of uniform illumination and homogenous mechanical properties. When a spherically focused transducer, or dynamically focused transducer array, is used as in traditional pulse-echo ultrasound imaging, one-dimensional images are calculated and properly aligned to form an image. ${ }^{6,7}$ To improve the lateral resolution and enlarge the imaging view, unfocused transducers are employed necessitating an advanced reconstruction method. Many approximate empirical algorithms were proposed in early studies, including weighted delay and sum ${ }^{8}$, the optimal statistical approach ${ }^{9}$ and radon transform in a far field approximation $^{10}$. Recently, a rigorous modified back projection reconstruction method for planar, cylindrical and spherical detecting geometries has been reported. ${ }^{11}$

Unlike traditional optical methods, which suffer from the high scattering of biological tissues, PAT combines the merits of sensitive absorbing contrast and high ultrasonic resolution at the sub-millimeter level. An imaging depth of several centimeters has also been demonstrated in tissue phantoms. ${ }^{12}$ Because of its unique capability to see the colorful nature of the life. PAT has been successfully applied in structural and functional imaging of the brain ${ }^{13}$ and depiction of tumor angiogenesis ${ }^{14}$ in small animal models in vivo. Several strong contrast agents for enhancing optical absorption have been demonstrated in in-vivo applications, such as indocyanine green (ICG) ${ }^{15}$, poly(ethylene glycol)-coated nanoshells ${ }^{16}$. Currently,

${ }^{*}$ Corresponding author. E-mail address: lwang@tamu.edu

Photons Plus Ultrasound: Imaging and Sensing 2006: The 7th Conference on Biomedical

Thermoacoustics, Optoacoustics, and Acousto-optics, edited by A. A. Oraevsky, et. al

Proc. of SPIE Vol. 6086, 60860Y (2006) • 1605-7422/06/\$15 • doi: 10.1117/12.646357

Proc. of SPIE Vol. 6086 60860Y-1 
many researchers are proceeding towards molecular imaging, e.g. visualizing tumors genetically marked by lacZ gene ${ }^{17}$.

An attractive advantage of PAT is that no obvious speckle has been seen. ${ }^{4}$ Optical speckle was first observed as granular patterns in the reflection of a laser beam from a rough surface. ${ }^{18}$ Later researchers realized that it was a fundamental feature of a series of coherent imaging modalities, including laser illuminator, pulse-echo ultrasound, synthetic aperture radar (SAR) and optical coherence tomography (OCT), etc. It is believed that speckle is mainly caused by the interference of coherent radiation from the rough microstructures of the object within a resolution cell. Furthermore, because light is usually detected as time-integrated intensity, temporal coherence is required to observe the speckle patterns in images formed by laser. Speckle was first considered as noise, because its texture is not directly related to the object and blurs the image. Various techniques have been developed to reduce speckles, for instance, spatial and frequency compounding, etc. However, further studies have revealed that speckle carries information about objects' microstructures. This fact has been applied in efforts at tissue characterization. ${ }^{19}$

To quantitatively understand the characteristics of speckle, a classic random walk model was proposed by Goodman. ${ }^{20}$ There, radiation from random sources were modeled as independent phasors, whose own magnitude and phases were independent, and the phases were uniformly distributed in $[-\pi, \pi)$. The images consisted of certain information extracted from the interference field in the imaging domain. Researchers are mainly interested in the first-order and the second-order properties of a speckle image. The former refers to the distribution of a single image point, while the latter represents the joint statistics of two image points. The image from the laser, represents the intensity of the interference field, following an exponential distribution at every single point. Burckhardt adopted this model to study speckle in pulse-echo ultrasound and found from the ultrasonic brightness images that physically the magnitude of the interference field is Rayleigh distributed. ${ }^{21}$ The autocorrelation of speckle, as a most important second-order statistical property, quantifies the average size of speckle and was found to relate primarily to the wavelength of the radiation and the systems' numerical aperture. ${ }^{20,22}$

From the view of imaging science, PAT belongs to a kind of coherent diffraction tomography, because its images are obtained by coherent reconstruction from detected photoacoustic waves. In this paper, we systematically studied the answer to the question of why no obvious speckle has been observed in PAT. First, a theoretical model of speckle in PAT was established based on linear system theory. Then, the reason for the lack of speckle in PAT was discussed in detail in comparison with other imaging modalities. Our theoretical explanations are validated by simulations.

\section{THEORETICAL MODEL}

Although a random walk model has been applied to study speckle in other imaging modalities that use pulsed radiation, like pulse-echo ultrasound, this may be confusing because phasor is not suitable for describing broadband pulses. Also, it does not directly relate the statistics of the speckle in an image to the microstructures of the object. Here, we model the speckle in PAT based on linear system theory to overcome the aforementioned problems.

PAT, similar to a variety of other imaging modalities comprising a series of linear operations, can be modeled as a linear system $L(\cdot)$, which maps an object $\mathbf{O}(\vec{r})$ into an image $\mathbf{M}(\vec{r})$. Both $\mathbf{O}(\vec{r})$ and $\mathbf{M}(\vec{r})$ are spatial stochastic processes in our study of speckle. An ideal system has a point spread function $\delta(\vec{r})$, which is a Dirac delta function, and can resolve any fine structure. However, this is not available in practice, mainly due to the fact that the limited bandwidth of detection and the imperfect reconstruction. $h(\vec{r})$ of real imaging systems which usually are high peaked at the origin and extend to cover a certain volume, called the resolution cell $V$, which we mentioned without an explicit definition above. $\mathrm{Xu}$ and Wang showed the PAT system with a band-limited point transducer has a spatially invariant $h(\vec{r})^{23}$ under perfect reconstruction. Thus, the imaging process can be expressed as:

$$
\mathbf{M}(\vec{r})=L[\mathbf{O}(\vec{r})]=\mathbf{O}(\vec{r}) * h(\vec{r}),
$$


where * represents the spatial convolution. By treating $h(\vec{r})$ as spatially partial waves radiating from rough microstructures of $\mathbf{O}(\vec{r}), \mathbf{M}(\vec{r})$ is the interference field of these waves.

Strictly speaking, the object function $\mathbf{O}(\vec{r})$ in PAT represents the initial pressure field $p_{0}$ after impulse excitation, given by:

$$
p_{0}(\vec{r})=\Gamma(\vec{r}) F(\vec{r}) \mu_{\mathrm{a}}(\vec{r}) .
$$

$\Gamma(\vec{r})$ is the Gröneisen parameter that, describes the object's mechanical properties; $F(\vec{r})$ is the local fluence rate; and $\boldsymbol{\mu}_{\mathbf{a}}(\vec{r})$ is the absorption coefficient. Like in many other studies, we assume $\Gamma(\vec{r})$ and $F(\vec{r})$ to be uniform in the area of interest here. We model the rough microstructures by randomly distributing a large number of i.i.d. (independent and identically distributed) absorbers in a resolution cell, which is usually a fraction of cubic millimeters, with complete spatial randomness (CSR). In other words, $\mathbf{O}(\vec{r})$ is a spatial Poisson point process which can be written as:

$$
\mathbf{O}(\vec{r})=\Gamma F \boldsymbol{\mu}_{\mathbf{a}}(\vec{r})=\Gamma F \sum_{i=1}^{\mathbf{N}} \mathbf{a}_{\mathbf{i}} \delta\left(\vec{r}-\overrightarrow{\mathbf{r}}_{i}\right),
$$

Where the $\mathrm{i}^{\text {th }}$ absorber is located at $\overrightarrow{\mathbf{r}}_{i}$ with absorption strength $\mathbf{a}_{i} . \mathbf{N}$ is a spatial Poisson process, whose intensity $\rho(\vec{r})$ is the local volume density of the absorbers. Denote the mean and variance of $\mathbf{a}$ as $a$ and $\sigma_{a}^{2}$, respectively. The mean and autocovariance of $\mathbf{O}(\vec{r})$ is shown in the Appendix to be:

$$
E(\mathbf{O}(\vec{r}))=\Gamma F a \rho(\vec{r})
$$

and

$$
C_{\mathbf{O}}\left(\vec{r}, \vec{r}^{\prime}\right)=\Gamma^{2} F^{2}\left(a^{2}+\sigma_{a}^{2}\right) \rho(\vec{r}) \delta\left(\vec{r}-\vec{r}^{\prime}\right) .
$$

In practice, absorbing objects always have limited volume. For simplicity of analysis, we consider that all of the absorbers are uniformly distributed in a bounded volume $V$, which can be modeled by taking $\rho(\vec{r})$ as:

$$
\rho(\vec{r})=\rho_{0} U_{V(\vec{r})}(\vec{r}),
$$

where $U_{V(\vec{r})}(\vec{r})$ is defined as:

$$
U_{V(\vec{r})}(\vec{r})=\left\{\begin{array}{ll}
1 & \vec{r} \in V \\
0 & \vec{r} \notin V
\end{array} .\right.
$$

Following the theory of linear systems, $\mathbf{M}(\vec{r})$ has the form of

$$
\mathbf{M}(\vec{r})=\Gamma F \sum_{i=1}^{\mathbf{N}} \mathbf{a}_{i} h\left(\vec{r}-\overrightarrow{\mathbf{r}}_{i}\right),
$$

which is a spatial shot noise process that is proved to converge to a normal distribution under the assumption of stationarity ${ }^{24}$, which is approximately satisfied in the center region of a big absorbing object. The expectation of $\mathbf{M}(\vec{r})$, the baseline shape of the image, is calculated to be:

$$
\begin{aligned}
E(\mathbf{M}(\vec{r})) & =E(L(\mathbf{O}(\vec{r})))=L(E(\mathbf{O}(\vec{r}))) \\
& =\Gamma F \int_{V\left(\vec{r}^{\prime}\right)} h\left(\vec{r}-\vec{r}^{\prime}\right) a \rho_{0} d V\left(\vec{r}^{\prime}\right) \\
& =\Gamma F a \rho_{0} \int_{V\left(\vec{r}^{\prime}\right)} h\left(\vec{r}-\vec{r}^{\prime}\right) d V\left(\vec{r}^{\prime}\right) .
\end{aligned}
$$


Although the integration of $h(\vec{r})$ over the whole space is usually zero due to the lack of DC components in the transducer response, the ensemble average of $\mathbf{M}(\vec{r})$ here is typically not zero, because the integral is over the limited volume $V\left(\vec{r}^{\prime}\right)$, unless $a$ is zero. The autocovariance of $\mathbf{M}(\vec{r})$ is given by:

$$
\begin{aligned}
C_{\mathbf{M}}\left(\vec{r}, \vec{r}^{\prime}\right) & =h(\vec{r}) * C_{\mathbf{O}}\left(\vec{r}, \vec{r}^{\prime}\right) * h\left(\vec{r}^{\prime}\right) \\
& =h(\vec{r}) * \Gamma^{2} F^{2}\left(a^{2}+\sigma_{a}^{2}\right) \rho(\vec{r}) \delta\left(\vec{r}-\vec{r}^{\prime}\right) * h\left(\vec{r}^{\prime}\right) \\
& =\Gamma^{2} F^{2}\left(a^{2}+\sigma_{a}^{2}\right) \rho\left(\vec{r}^{\prime}\right) h\left(\vec{r}-\vec{r}^{\prime}\right) * h\left(\vec{r}^{\prime}\right) \\
& =\Gamma^{2} F^{2}\left(a^{2}+\sigma_{a}^{2}\right) \rho_{0} \int_{V\left(\vec{r}^{\prime \prime}\right)} h\left(\vec{r}-\vec{r}^{\prime \prime}\right) h\left(\vec{r}^{\prime}-\vec{r}^{\prime \prime}\right) d^{3} \vec{r}^{\prime \prime}
\end{aligned}
$$

The variance of $\mathbf{M}(\vec{r})$, which depicts the fluctuation of the speckle pattern with respect to the baseline, can be written as:

$$
\begin{aligned}
& \operatorname{Var}(\mathbf{M}(\vec{r}))=C_{\mathbf{M}}(\vec{r}, \vec{r}) \\
& \quad=\Gamma^{2} F^{2}\left(a^{2}+\sigma_{a}^{2}\right) \rho_{0} \int_{V\left(\vec{r}^{\prime}\right)} h^{2}\left(\vec{r}-\vec{r}^{\prime}\right) d^{3} \vec{r}^{\prime} .
\end{aligned}
$$

Now, we define a criterion, speckle contrast $C r_{\text {speckle }}$, as the standard deviation of a speckle image point over its mean which quantifies the visibility of the speckle pattern in an image. In PAT, it can be obtained easily from (9) and (11):

$$
\begin{aligned}
C r_{\text {speckle }} & =\frac{\sqrt{\operatorname{Var}(\mathbf{M}(\vec{r}))}}{E(\mathbf{M}(\vec{r}))} \\
& =\sqrt{1+\frac{\sigma_{a}^{2}}{a^{2}}} \frac{1}{\sqrt{\rho_{0}}} \frac{\sqrt{\int_{V\left(\vec{r}^{\prime}\right)} h^{2}\left(\vec{r}-\vec{r}^{\prime}\right) d^{3} \vec{r}^{\prime}}}{\int_{V\left(\vec{r}^{\prime}\right)} h\left(\vec{r}-\vec{r}^{\prime}\right) d^{3} \vec{r}^{\prime}} .
\end{aligned}
$$

In other words, the visibility of a speckle pattern is related to the fluctuating level of the absorption strength, the volume density of the absorbers and a system-dependent factor associated with the macroscopic shape of the absorbing structure.

\section{SIMULATION METHODOLOGY}

In the following section, we will extensively discuss the reason responsible for the lack of speckle in PAT that is implied by (13), and validated by simulation. In our simulation, $\mathrm{N}$ point absorbers with random positive strengths are placed in volume $\mathrm{V}$ by randomly choosing the $\mathrm{x}, \mathrm{y}, \mathrm{z}$ coordinates under an uniform distribution, to form an object function $O(x, y, z)$. Given the transducer's receiving response $H_{b}(\widetilde{k})$ :

$$
H_{b}(\tilde{k})=4\left(0.5+0.5 \cos \left(\pi \frac{\tilde{k}}{2 \widetilde{k}_{c}}\right)\right)\left(0.5-0.5 \cos \left(\pi \frac{\tilde{k}}{2 \widetilde{k}_{c}}\right)\right),
$$

which is bell-shaped, mimicking the real situation. The system's one-dimensional point spread function $h(r)$ is calculated by the following numerical integration ${ }^{23}$ :

$$
h(r)=\frac{1}{2 \pi^{2}} \int_{0}^{\infty} H_{b}(\tilde{k}) j_{0}(\tilde{k} r) \tilde{k}^{2} d \tilde{k} .
$$


Here, acoustic wavenumber $\widetilde{k}_{c}=\frac{2 \pi f_{c}}{c}\left(f_{c}\right.$ is the center frequency of the transducer; $c$ is the velocity of the sound.); $h(r)$ is then regridded to space obtaining $h(x, y, z)$. Image $I(x, y, z)$ is obtained by convolving $O(x, y, z)$ and $h(x, y, z)$ by $I=\operatorname{IFFT}\{F F T(O) F F T(h)\}$, which significantly reduces the calculation time.

\section{DISCUSSION}

A typical simulation result is shown in Figure 1 where 0.15 million point absorbers form a spherical absorbing region with a diameter of $5 \mathrm{~mm}$, corresponding to a low volume density of about $2.3 \times 10^{3} / \mathrm{mm}^{3}$. A PAT image was taken at a central plane $z=0$. (a) shows the object function in this plane. The transducer center frequency is set to $2.25 \mathrm{MHz}$, which is used in the real PAT system in our laboratory. (b) is the resultant one-dimensional $h(r)$, whose main lobe extends to about $0.6 \mathrm{~mm}$. (c) shows the PAT image where the granular speckle pattern is visible, which is strong at the edge of the absorbing region due to the band-limited nature of the PAT system. By assuming that ergodicity holds, the distribution of $\mathbf{M}(\vec{r})$ near the center is approximated by studying the statistics of image values within a $2.5 \mathrm{~mm} \times 2.5 \mathrm{~mm}$ central area and is found to follow a normal distribution well in (d), which meets our prediction above.

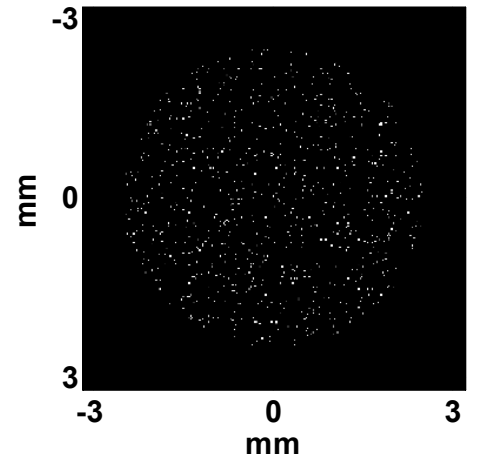

(a)

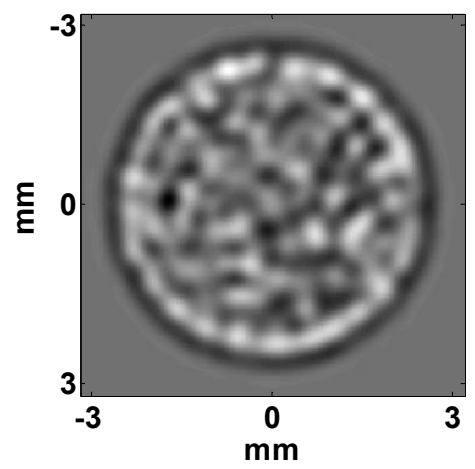

(c)

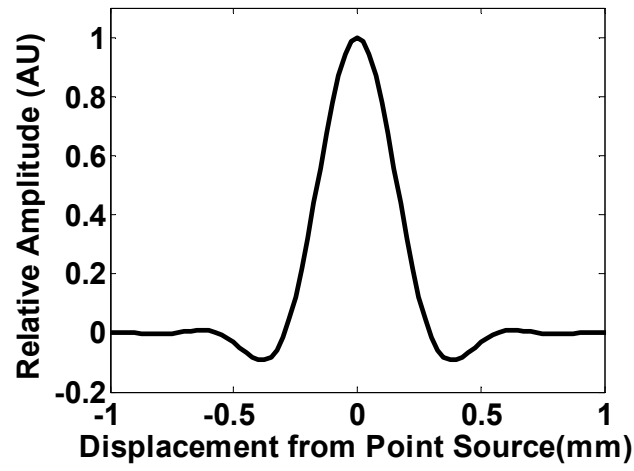

(b)

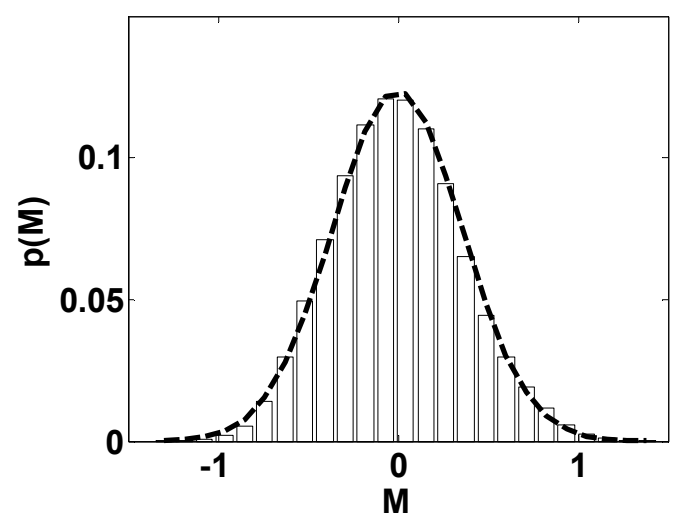

(d)

Figure 1. (a) Central plane of a 5mm diameter absorbing sphere including 150,000 absorbers. (b) One dimensional point spread function of PAT system with $2.25 \mathrm{MHz}$ transducer; (c) Simulated PAT image with "speckle"; (d) The distribution of $\mathbf{M}(\vec{r})$ near the center of image (bar) compared to a normal probability density function (dash line). 
What's more, the speckle contrast in PAT is found to be inversely proportional to the square root of the absorbers' volume density. Figure 2 validates this. (b) is the PAT image of an absorbing object containing 10 times more absorbers than (a). The speckle pattern obviously tends to less visible in (b). In a quantitative study, we calculated the speckle contrast for images of objects with five different volume densities based on the definition given in (12), and found that the change in speckle contrast follows the inverse square root relation well in (c). To link this simulation with a real situation, consider a dye solution with a concentration at $1 \mu$ Molar. The volume density of the dye molecules will be on the order of $10^{14} / \mathrm{mm}^{3}$, which is more than eight orders higher than the highest volume density in our simulation corresponding to (b). Thus, the visibility of the speckle will be over 10,000 times less than (b).

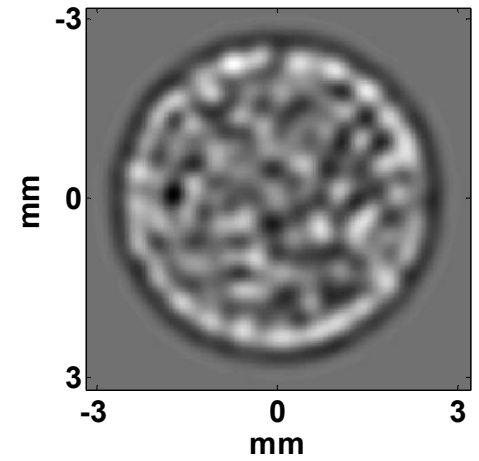

(a)

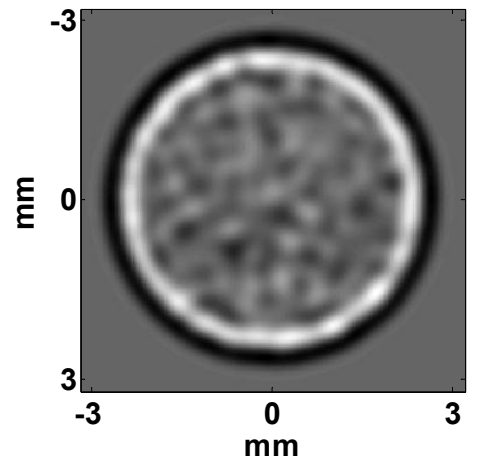

(b)

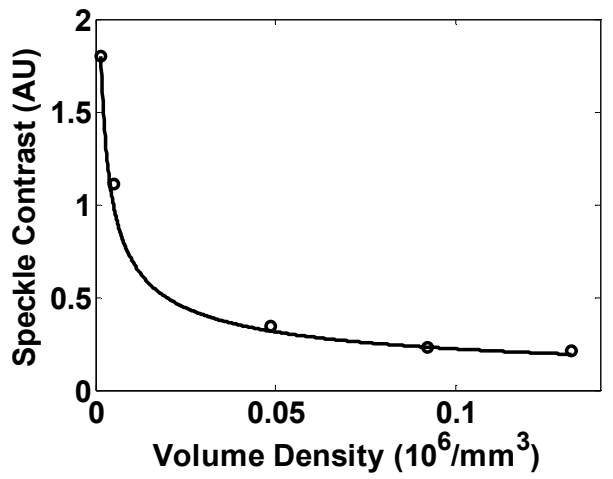

(c)

Figure 2. Simulated PAT image of the central plane of a 5mm diameter sphere including (a) 150,000 (b) $1,500,000$ absorbers. (c) Profile of speckle contrast in PAT image vs. absorber density (circle) compared with theoretical prediction (solid line).

It is also interesting to compare the PAT image with an ultrasonic radiofrequency image. Both systems are linear. For the ultrasonic radiofrequency image, the results in section 2 should also hold. But the object function of the ultrasonic imaging represents a change in acoustic impedance, which is a zero-mean process in soft tissues under good approximation. It follows that the denominator in (12) will be zero everywhere due to local cancellation and the speckle contrast will approach infinity. Physically, the fluctuations of the speckle pattern will fill the whole dynamic range. On the contrary, the object function of PAT is positive everywhere, and hence the baseline with a high edge will suppress the visibility of the speckle pattern. Figure 3 shows the effects of different kinds of object function. (a) is the image of all positive object 
function corresponding to the case of PAT, while (b) is an image of a zero-mean object function, mimicking the case of the ultrasonic radiofrequency image. Speckle is much more obvious in the latter. Here, for fairness of comparison, we use the same $h(\vec{r})$ for both. In the real situation, $h(\vec{r})$ of the ultrasonic imaging system has an anisotropic shape and the speckle pattern is more shuttle-shaped.

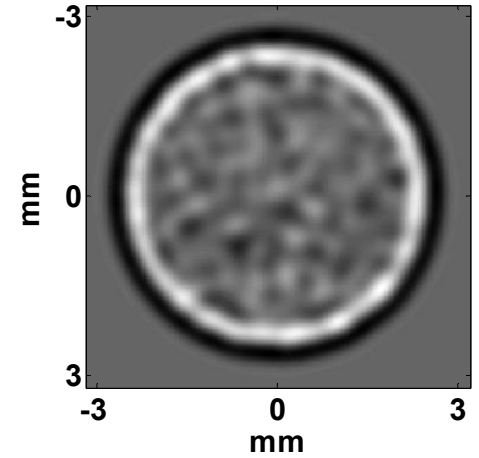

(a)

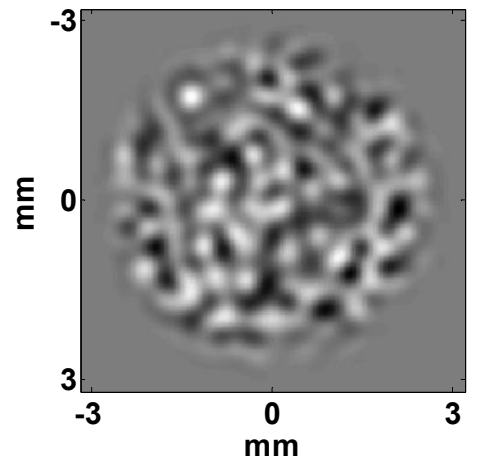

(b)

Figure 3. Simulated image of the central plane of a $5 \mathrm{~mm}$ diameter sphere including $1,500,000$ absorbers with (a) positive-mean and (b) zero-mean object function.

\section{CONCLUSION}

In summary, we systemically studied the speckle in PAT for the first time. A theoretical expression for speckle contrast in PAT, a quantitative standard for the visibility of the speckle in an image, was obtained based on linear system theory. With minor changes, this method can be extended to study other linear imaging systems as well. The lack of speckle in PAT is mainly due to the positive object function and the inverse square root dependence of speckle contrast on the absorbers' volume density. The established model of speckle is closely related to microscopic information about the absorbing objects, such as the absorbers' volume density and absorbing strength. For potential future exploitation of these findings in tissue characterization, the expression of speckle contrast can serve as a guideline for designing systems that maximize the visibility of speckle, for example, by choosing the right transducer; a basis of developing schemes to extract this kind of microscopic information and a tool for understanding the limitations. Also, clustered particles can be taken into consideration by introducing more advanced spatial processes, like the Cox process.

\section{ACKOWLEDGEMENTS}

This project is sponsored by the National Institutes of Health grants R01 NS46214 and R01 EB000712.

\section{APPENDIX}

Full specification of the statistics of $\mathbf{O}(\vec{r})$ in (3) requires knowledge of the joint distributions of all of the random variables there: $\mathbf{N}, \mathbf{a}_{i}, \overrightarrow{\mathbf{r}}_{i}(i=1,2, \cdots, \mathbf{N})$, which are mutually independent based on our physical model. We are interested in the mean and autocovariance of $\mathbf{O}(\vec{r})$. The results of the point 
process consisting of identical points with unit strength, i.e. $\mathbf{a}_{i} \equiv 1$, are given by Barrett ${ }^{25}$. Here we extend it to the case of points with randomized strengths.

Following a general procedure for dealing with the summation of a random number of items, we start with the statistics of $\mathbf{O}(\vec{r})$ conditional on $\mathbf{N}$. After that, the statistics of $\mathbf{O}(\vec{r})$ are obtained by considering a marginal distribution over $\mathbf{N}$.

$$
\begin{aligned}
& E\{\mathbf{O}(\vec{r}) \mid \mathbf{N}\} \\
= & \Gamma F \int_{V} d^{3} \overrightarrow{\mathbf{r}}_{1} \int_{V} d^{3} \overrightarrow{\mathbf{r}}_{2} \cdots \int_{V} d^{3} \overrightarrow{\mathbf{r}}_{\mathbf{N}} \int_{a} d \mathbf{a}_{1} \int_{a} d \mathbf{a}_{2} \cdots \int_{a} d \mathbf{a}_{\mathbf{N}} \operatorname{pr}\left(\left\{\overrightarrow{\mathbf{r}}_{i}\right\} \mid \mathbf{N}\right) \operatorname{pr}\left(\left\{\mathbf{a}_{i}\right\} \mid \mathbf{N}\right) \sum_{i=1}^{\mathbf{N}} \mathbf{a}_{i} \delta\left(\vec{r}-\overrightarrow{\mathbf{r}}_{i}\right) \\
= & \Gamma F \sum_{i=1}^{\mathbf{N}} \int_{V} d^{3} \overrightarrow{\mathbf{r}}_{i} \int_{a} d \mathbf{a}_{i} \operatorname{pr}\left(\overrightarrow{\mathbf{r}}_{i} \mid \mathbf{N}\right) \operatorname{pr}\left(\mathbf{a}_{i} \mid \mathbf{N}\right) \mathbf{a}_{i} \delta\left(\vec{r}-\overrightarrow{\mathbf{r}}_{i}\right) \\
= & \Gamma F a \mathbf{N} p r(\overrightarrow{\mathbf{r}} \mid \mathbf{N}) .
\end{aligned}
$$

Throughout this paper, we use $\operatorname{pr}(\cdot)$ to denote the probability density function. Following the definition of the Poisson point process, $\operatorname{pr}(\overrightarrow{\mathbf{r}} \mid \mathbf{N})$ is independent of $\mathbf{N}$ and can be written as:

$$
\operatorname{pr}(\overrightarrow{\mathbf{r}} \mid \mathbf{N})=\operatorname{pr}(\overrightarrow{\mathbf{r}})=\frac{\rho(\vec{r})}{\int_{V} d^{3} \vec{r} \rho(\vec{r})}
$$

and

$$
\overline{\mathbf{N}}=\int_{V} d^{3} \vec{r} \rho(\vec{r})
$$

So the mean of $\mathbf{O}(\vec{r})$ is

$$
\begin{aligned}
E(\mathbf{O}(\vec{r}))= & <\Gamma F \mathbf{N} \operatorname{apr}(\overrightarrow{\mathbf{r}} \mid \mathbf{N})>_{\mathbf{N}} \\
= & \Gamma F a \frac{\rho(\vec{r})}{\int_{V} d^{3} \vec{r} \rho(\vec{r})} \overline{\mathbf{N}} \\
& =\Gamma F a \rho(\vec{r}) .
\end{aligned}
$$

The autocorrelation function of $\mathbf{O}(\vec{r})$ is defined by:

$$
R_{\mathbf{O}}\left(\vec{r}, \vec{r}^{\prime}\right)=E\left\{\mathbf{O}(\vec{r}) \mathbf{O}\left(\vec{r}^{\prime}\right)\right\} .
$$

$E\left\{\mathbf{O}(\vec{r}) \mathbf{O}\left(\vec{r}^{\prime}\right) \mid \mathbf{N}\right\}$

$=\Gamma^{2} F^{2} \int_{V} d^{3} \overrightarrow{\mathbf{r}}_{1} \int_{V} d^{3} \overrightarrow{\mathbf{r}}_{2} \cdots \int_{V} d^{3} \overrightarrow{\mathbf{r}}_{\mathbf{N}} \int_{a} d \mathbf{a}_{1} \int_{a} d \mathbf{a}_{\mathbf{2}} \cdots \int_{a} d \mathbf{a}_{\mathbf{N}} p r\left(\left\{\overrightarrow{\mathbf{r}}_{i}\right\} \mid \mathbf{N}\right) \operatorname{pr}\left(\left\{\mathbf{a}_{i}\right\} \mid \mathbf{N}\right) \sum_{i=1}^{\mathbf{N}} \mathbf{a}_{i} \delta\left(\vec{r}-\overrightarrow{\mathbf{r}}_{i}\right) \sum_{i=j}^{\mathbf{N}} \mathbf{a}_{j} \delta\left(\vec{r}^{\prime}-\overrightarrow{\mathbf{r}}_{j}\right)$

$=\Gamma^{2} F^{2}\left\{\sum_{i=1}^{\mathbf{N}} \int_{V} d^{3} \overrightarrow{\mathbf{r}}_{1} \int_{V} d^{3} \overrightarrow{\mathbf{r}}_{2} \cdots \int_{V} d^{3} \overrightarrow{\mathbf{r}}_{\mathbf{N}} \int_{a} d \mathbf{a}_{1} \int_{a} d \mathbf{a}_{2} \cdots \int_{a} d \mathbf{a}_{\mathbf{N}} \operatorname{pr}\left(\overrightarrow{\mathbf{r}}_{i} \mid \mathbf{N}\right) \operatorname{pr}\left(\mathbf{a}_{i} \mid \mathbf{N}\right) \mathbf{a}_{i}^{2} \delta\left(\vec{r}-\overrightarrow{\mathbf{r}}_{i}\right) \delta\left(\vec{r}^{\prime}-\overrightarrow{\mathbf{r}}_{i}\right)\right.$

$\left.+\sum_{i=1}^{\mathbf{N}} \sum_{j \neq i, j=1}^{\mathbf{N}} \mathbf{a}_{j} \int_{V} d^{3} \overrightarrow{\mathbf{r}}_{1} \int_{V} d^{3} \overrightarrow{\mathbf{r}}_{2} \cdots \int_{V} d^{3} \overrightarrow{\mathbf{r}}_{\mathbf{N}} \int_{a} d \mathbf{a}_{1} \int_{a} d \mathbf{a}_{2} \cdots \int_{a} d \mathbf{a}_{\mathbf{N}} \operatorname{pr}\left(\overrightarrow{\mathbf{r}}_{i}, \overrightarrow{\mathbf{r}}_{j} \mid \mathbf{N}\right) \operatorname{pr}\left(\mathbf{a}_{i}, \mathbf{a}_{j} \mid \mathbf{N}\right) \mathbf{a}_{i} \mathbf{a}_{j} \delta\left(\vec{r}-\overrightarrow{\mathbf{r}}_{i}\right) \delta\left(\vec{r}^{\prime}-\overrightarrow{\mathbf{r}}_{j}\right)\right\}$

$=\Gamma^{2} F^{2}\left\{\mathbf{N} p r\left(\overrightarrow{\mathbf{r}}_{i} \mid \mathbf{N}\right)\left(a^{2}+\sigma_{a}^{2}\right) \delta\left(\vec{r}-\vec{r}^{\prime}\right)+\left(\mathbf{N}^{2}-\mathbf{N}\right) a^{2} p r\left(\overrightarrow{\mathbf{r}}, \overrightarrow{\mathbf{r}}^{\prime} \mid \mathbf{N}\right)\right\}$.

The autocovariance of $\mathbf{O}(\vec{r})$ is given by: 


$$
\begin{aligned}
& C_{\mathbf{O}}\left(\vec{r}, \vec{r}^{\prime}\right)=R_{\mathbf{O}}\left(\vec{r}, \vec{r}^{\prime}\right)-E(\mathbf{O}(\vec{r})) E\left(\mathbf{O}\left(\vec{r}^{\prime}\right)\right) . \\
& =\Gamma^{2} F^{2}\left\{\mathbf{N} p r\left(\overrightarrow{\mathbf{r}}_{i} \mid \mathbf{N}\right)\left(a^{2}+\sigma_{a}^{2}\right) \delta\left(\vec{r}-\vec{r}^{\prime}\right)+\left(\mathbf{N}^{2}-\mathbf{N}\right) a^{2} p r\left(\overrightarrow{\mathbf{r}}_{i}, \overrightarrow{\mathbf{r}}_{j} \mid \mathbf{N}\right)-a^{2} \rho(\vec{r}) \rho\left(\vec{r}^{\prime}\right)\right\} .
\end{aligned}
$$

Again, following the definition of the Poisson point process, we have:

$$
\begin{aligned}
\operatorname{pr}\left(\overrightarrow{\mathbf{r}}, \overrightarrow{\mathbf{r}}^{\prime} \mid \mathbf{N}\right) & =\operatorname{pr}(\overrightarrow{\mathbf{r}} \mid \mathbf{N}) \operatorname{pr}\left(\overrightarrow{\mathbf{r}}^{\prime} \mid \mathbf{N}\right)=\operatorname{pr}(\overrightarrow{\mathbf{r}}) \operatorname{pr}\left(\overrightarrow{\mathbf{r}}^{\prime}\right), \\
& =\frac{\rho(\vec{r}) \rho\left(\vec{r}^{\prime}\right)}{\int_{V} d^{3} \vec{r} \rho(\vec{r}) \int_{V} d^{3} \vec{r}^{\prime} \rho\left(\vec{r}^{\prime}\right)},
\end{aligned}
$$

And

$$
<\mathbf{N}^{2}-\mathbf{N}>_{\mathbf{N}}=\overline{\mathbf{N}}^{2}
$$

Substitute into (A.6), we have:

$$
C_{\mathbf{o}}\left(\vec{r}, \vec{r}^{\prime}\right)=\Gamma^{2} F^{2}\left(a^{2}+\sigma_{a}^{2}\right) \rho(\vec{r}) \delta\left(\vec{r}-\vec{r}^{\prime}\right)
$$

The above conclusion can be generalized to $\vec{r} \in R^{n}$ and directly reduces to the result in a onedimensional generalized Poisson point process, or shot noise ${ }^{26}$.

\section{REFERENCES}

1. A. Rosencwaig, "Photoacoustic spectroscopy," Ann. Rev. Biophys. Bioeng. 9, 31-54(1980).

2. R. A. Kruger, P.Liu, Y. R. Fang and C. R. Appledom, "Photoacoustic ultrasound (PAUS) reconstruction tomography," Med. Phys., 22, 1605-1609 (1995).

3. X. Wang, Y. Xu, M. Xu, S.Yokoo, E. S. Fry, "Photoacoustic tomography of biological tissues with high cross-section resolution: reconstruction and experiment," Med. Phys., 29, 2799-2805 (2002).

4. L. V. Wang, "Ultrasound-mediated biophotonic imaging: a review of acousto-optical tomography and photo-acoustic tomography," Disease Markers, 19,123-128 (2002).

5. V. E., Gusev and A. A. Karabutov, "Laser optoacoustics," American Institute of Physics (1992).

6. G. Ku and L. V. Wang, "Thermoacoustic tomography in biological tissues," Med. Phys., 28, 1958-1963 (2001).

7. B. Yin, D. Xing, Y. Wang, Y. Zeng, Y. Tan and Q. Chen, "Fast photoacoustic imaging system based on 320-element linear transducer array," Phys. Med. Biol., 49, 1339-1346(2004).

8. C. G. A. Hoelen and F. F. M., DeMul "Image reconstruction for photoacoustic scanning of tissue structures," Appl. Opt., 39(31), 5872-5883 (2000).

9. Y. V. Zhulina, "Optimal statistical approach to optoacoustic image reconstruction," Appl. Opt., 39(32),5971-5977 (2000).

10 P. Y. Liu, "The P-transform and photoacoustic image reconstruction," Phys. Med. Biol., 43, 667-674 (1998).

11. M. Xu, Y. Xu and L. V. Wang, "Time-domain reconstruction algorithms and numerical simulations for thermoacoustic tomography in various geometries," IEEE Trans. Med. Imaging, 50(9), 1086-1099 (2003).

12. G. $\mathrm{Ku}$ and L. V. Wang, "Deeply penetrating photoacoustic tomography in biological tissues enhanced with an optical contrast agent," Opt. Lett., 30(5), 507-509 (2005).

13. X. Wang, Y. Pang, G. Ku, X. Xie, G. Stoica and L. V. Wang, "Non-invasive laser-induced photoacoustic tomography for structural and functional imaging of the brain in vivo," Nat. Biotech. 21(7), 803-806 (2003).

14. G. Ku, X. Wang, X. Xie, G. Stoica and L. V. Wang, "Imaging of tumor angiogenesis in rat brains in vivo by photoacoustic tomography," Appl. Opt., 44(5), 770-775 (2005).

15. X. Wang, G. Ku, M. A. Wegiel, D. J. Bornhop, G. Stoica and L. V. Wang, "Non-invasive photoacoustic angiography of animal brains in vivo with NIR light and an optical contrast agent," Opt. Lett. 29(7), 730 732 (2004). 
16. Y. Wang, X. Xie, X. Wang, G. Ku, K. L. Gill, D. P. O'Neal, G. Stoica and L. V. Wang, "Photoacoustic tomography of a nanoshell contrast agent in the in vivo rat brain," Nano Lett., 4(9), 1689-1692 (2004).

17. L. Li, R. J. Zemp, Gina Lungu, G. Stoica and L. V. Wang, "Imaging of gene expression in vivo with photoacoustic tomography," Proc. SPIE Photons Plus Ultrasound: Imaging and Sensing, in press (2006).

18. J. D. Rigden and E. I. Gordon, "Granularity of scattered optical maser light," Proc. IRE 50, 6564-6574 (1962).

19. R. F. Wagner, M. F. Insana and D.G. Brown, "Unified approach to the detection and classification of speckle texture in diagnostic ultrasound," Opt. Eng., 25(6), 738-742 (1975).

20. J. W. Goodman, "Statistical properties of laser speckle patterns," in Laser Speckle and Related Phenomena edited by J. C. Dainty, Springer-Verlag (1984).

21. C. B. Burckhardt, "Speckle in ultrasound B-scans," IEEE Trans. Son. Ultrason., SU-25(1), 1-6 (1978).

22. R. F. Wagner, S. W. Smith, J. M. Sandrik and H. Lopez, "Statistics of speckle in ultrasound B-scans," IEEE Trans. Son. Ultrason., 30(3), 156-163 (1983).

23. M. Xu and L. V. Wang, "Analytic explanation of spatial resolution related to bandwidth and detector aperture size in thermoacoustic or photoacoustic reconstruction," Phys. Rev. E, 67, 056605 (2003).

24. L. Heinrich and V. Schmidt, "Normal convergence of multidimensional shot noise and rates of this convergence," Adv. Appl. Prob., 17,709-730 (1985).

25. H. H. Barrett and K. J. Mayers, "Foundations of Image Science," John Wiley\& Sons, 649-655 (2004).

26. A. Papoulis, "Probability, Random Variables and Stochastic Processes," McGraw-Hill, 359-362 (1991). 